\title{
Too Hot to Handle: An Evaluation of the Effect of Thermal Visual Representation on User Grasping Interaction in Virtual Reality
}

\author{
Andreea Dalia Blaga \\ DMT Lab, Birmingham City \\ University \\ Birminghan, United Kingdom \\ andreea.blaga@mail.bcu.ac.uk
}

\author{
Maite Frutos-Pascual \\ DMT Lab, Birmingham City \\ University \\ Birminghan, United Kingdom \\ maite.frutos@bcu.ac.uk \\ Ian Williams \\ DMT Lab, Birmingham City \\ University \\ Birminghan, United Kingdom \\ ian.williams@bcu.ac.uk
}

\author{
Chris Creed \\ DMT Lab, Birmingham City \\ University \\ Birminghan, United Kingdom \\ chris.creed@bcu.ac.uk
}

\begin{abstract}
Influence of interaction fidelity and rendering quality on perceived user experience have been largely explored in Virtual Reality (VR). However, differences in interaction choices triggered by these rendering cues have not yet been explored. We present a study analysing the effect of thermal visual cues and contextual information on 50 participants' approach to grasp and move a virtual mug. This study comprises 3 different temperature cues (baseline empty, hot and cold) and 4 contextual representations; all embedded in a VR scenario. We evaluate 2 different hand representations (abstract and human) to assess grasp metrics. Results show temperature cues influenced grasp location, with the mug handle being predominantly grasped with a smaller grasp aperture for the hot condition, while the body and top were preferred for baseline and cold conditions.
\end{abstract}

\section{Author Keywords}

Virtual Reality, Grasping Metrics, Hand Tracking, Hand Interaction

\section{CCS Concepts}

-Human-centered computing $\rightarrow$ Interaction design theory, concepts and paradigms; Virtual reality; Gestural input; Human computer interaction (HCI); User studies;

\footnotetext{
Permission to make digital or hard copies of all or part of this work for personal or classroom use is granted without fee provided that copies are not made or distributed for profit or commercial advantage and that copies bear this notice and the full citation on the first page. Copyrights for components of this work owned by others than the author(s) must be honored. Abstracting with credit is permitted. To copy otherwise, or republish, to post on servers or to redistribute to lists, requires prior specific permission and/or a fee. Request permissions from permissions@acm.org.

CHI'20, April 25-30, 2020, Honolulu, HI, USA

(C) 2020 Copyright held by the owner/author(s). Publication rights licensed to ACM ISBN 978-1-4503-6708-0/20/04 . .\$15.00

DOI: https : //doi .org/10.1145/3313831.3376554
}

\section{INTRODUCTION}

Immersive headsets have experienced an unprecedented growth in consumer availability [60], with companies such as Facebook, Microsoft or Samsung enabling accessible VR experiences for the masses. The sector's growth prospects reflect its potential to reshape the way people communicate $[64,91]$, play [43], work [45, 50] and learn [38, 107], achieving high levels of immersion [10].

Intuitive interaction in immersive environments plays a key role in increasing presence [36] which in turn can improve the overall user quality of experience. Seamless interactive VR systems often rely on hand interaction due to their multiple degrees of freedom [78] and human ability to use hands for acquiring and manipulating objects [2, 41, 63, 94]. Previous studies have shown that bare hand interaction (i.e. without the use of supplementary wearables) is linked to ease of access and naturalness. This is due to the absence of constraints imposed by wearable devices and its potential in delivering natural, intuitive and effective interaction in immersive environments [37, 49, 56, 73, 97]. When creating new bare hand interactive systems, several studies rely on predefined sets of gestures for natural interaction [22, 103, 104]. These interactions have predominantly been designed by researchers for optional recognition rather than for naturalness, being often arbitrary and not intuitive enough [66]. Therefore, one of the interaction challenges in VR is to improve the intuitive nature of interaction by improving natural grasping quality [67], thus mimicking the behaviour our human hands have in real environments [88].

Previous literature has explored the essential influencing factors to grasping real objects, with these being texture, hardness, weight and temperature/thermal conditions [5, 46]. Rendered textures and perceived hardness of objects and their effect on usability and user perception have been widely explored in immersive systems, with authors exploring texture repre- 


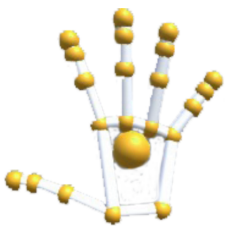

(a) Abstract

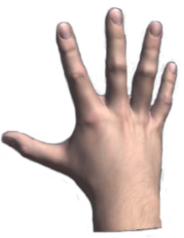

(b) Human

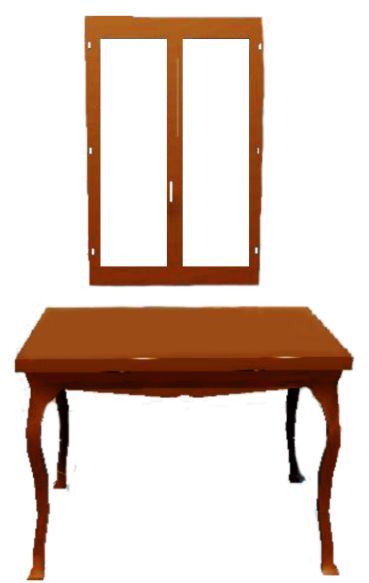

(c) VR Scenario
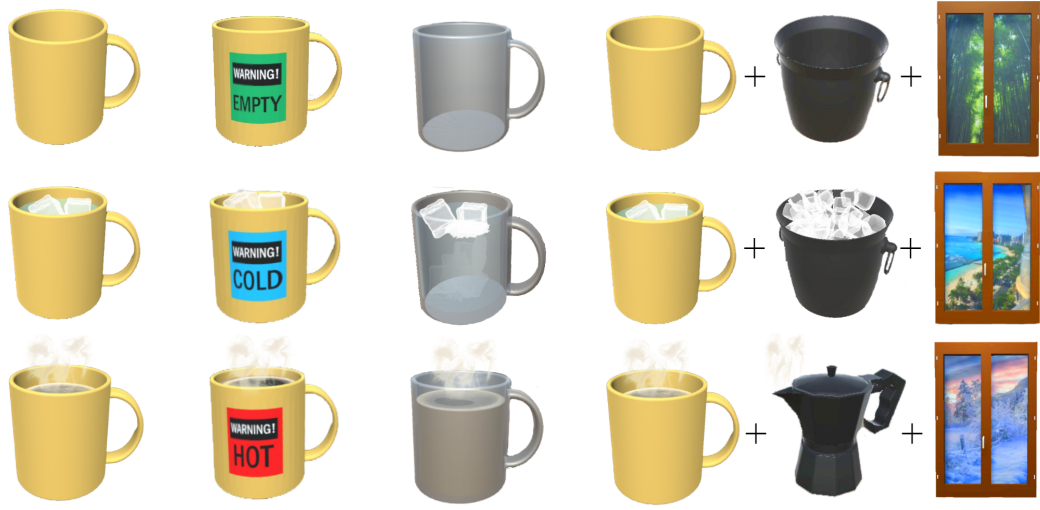

(d) Basic

(e) Label

(f) Glass

(g) Context Objects

Figure 1. Conditions under study, with 1(a) and 1(b) showcasing the human hand representations and 1(d), 1(e), 1(f) and 1(g) showcasing the VR environment conditions alongside visual thermal cues.

sentations using “custom-made” haptic actuators [5, 7, 32]. Additionally, thermal haptic feedback has been used to supplement immersive environments. Recent studies have applied thermal haptic feedback in differing configurations, namely, around the wrist [61], to augment emotions [89], show directional cues for users [62], guide user behaviour [90] and evaluate users' perception $[5,108]$. However, the effect of visual thermal cues for grasping interaction in VR has not been explored.

We present the first study evaluating thermal cues and their effect on grasp metrics in VR. Across 50 participants we report on user grasp aperture, grasp type and grasp location on the object under different visual thermal cues. We further evaluate four different contextual representations of the virtual environment to assess the influence of thermal visual feedback on grasp interaction. Findings from this study can be used as guidelines for interaction design, helping developers and designers to select the most suitable visual cues for facilitating an intuitive interaction with virtual objects and support improved natural interaction design for VR.

\section{RELATED WORK}

\section{Hand Interaction in VR}

Hand interactions in VR were initially developed using instrumented gloves [29], which inspired the development of wearable equipment such as wireless data gloves [44], markers [94] and electromyographic armbands [96, 106]. Wearable devices have been widely used for hand interaction methods, however, they inherently constrain human motion [101] and they have been often linked to discomfort [87], time-consuming configuration and problems with wider user adaptation [35].

As a result of these constraints, researchers have considered systems that do not require the full instrumentation of the hand [41] or devices that allow bare hand interaction [2, 34, 103]. Hand based interaction that enables users to manipulate objects as if they were real is known as grasping [3, 16]. This natural interaction with objects in VR plays an important role in increasing immersion in virtual environments [36], however is still one of the ongoing interaction challenges in VR [67].

\section{Grasping}

Researchers have investigated human's approach to grasping real objects, aiming at understanding certain aspects of human hand usage $[72,79]$, as well as classifying grasps in a discrete set of types [21, 28, 39]. However, this knowledge cannot be applied directly to virtual grasping, as it has been identified that users interact with virtual objects differently than they interact with real objects [54, 92, 98]. Therefore, virtual grasping has extensively been explored as a technical and computational challenge $[12,13,88]$.

It has been identified that object characteristics as shape and size influence the grasp choice when grasping real objects [25], as well as virtual objects [3, 4]. However, VR commonly lacks physical sensations of friction and weight to the hand [12], key constraints considered in grasping real objects.

\section{Visual Representations}

The main feedback cue in VR environments is the visual rendering [19]. Therefore, a number of works take advantage of the easiness with which visual cues can be controlled and evaluated in VR.

\section{User Representation}

The use of avatars and avatar representation in VR has received significant attention from the research community exploring how it effects the sense of body ownership [59, 84] and agency [40] as they allow users to locate their own body pose within the virtual environment.

Furthermore, additional visual aspects such as human-likeness $[48,52]$, gender [82] or transparency [15, 42] of the virtual representation have shown to influence ownership illusion as well as user performance.

The human hand is a powerful physical tool through which people interact with the surrounding world [30]. It has been identified that perceived naturalness of virtual hands can have 
a significant effect on perceived user presence [82] as well as own-body perception and immersion [48].

\section{Environment Representation}

One important facilitator of immersion in VR is fidelity, the degree of accuracy with which a system recreates real-world experiences $[14,20,55,100]$, with high levels of interaction fidelity being preferred for virtual object manipulation [75]. Multi-sensory feedback has proved to generate high levels of fidelity in VR [23, 32, 33, 80], with an increased number of works using haptic feedback devices to stimulate other sensory channels $[1,7,51,58,95,105]$. However, providing haptic feedback generally requires complex hardware, while still being limited [18, 47].

As an alternative, researchers rely on the concept of the kinesthetic visual capture [86], the dominance of vision over proprioception, to efficiently deceive user experience through visual cues in the environment [65].

Rietzler et al. [74] used visual cues to induce the haptic sensation of weight. They used perceivable tracking offsets of the virtual hand, nudging the user to lift the arm higher to perceive some form of additional exertion. To create the illusion of resistance of wind in VR, Pusch et al. [70, 71] used visual hand displacements. Their results show that the majority of participants felt a force that was pushing their hands. Rosas et al. [77] investigated how different types of visual cues for textures change depth perception. They found that textures with a pseudo-random distribution of circles provide the highest reliability in discriminating the distance of objects in motion. Biocca et al. [11] investigated sensory illusions in a virtual environment, and identified that when manipulating the visual analogue for a physical force, a virtual spring, users reported haptic sensations of "physical resistance", even though the interface included only visual representations. Vigier et al. [93] studied the role of visual cues (sky aspect, shadows, sun location and light effects) on climate perception (season, daytime and temperature) in virtual urban environments. Their results prove the feasibility of suggesting complex climatic perceptions and thermal feelings using just visual representations.

Although the effect of visual representations of the virtual environment on human perception has been investigated, its effect on wider interaction patterns such as grasp choice in VR is unknown.

\section{STUDY DESIGN}

\section{Apparatus}

We built a custom experimental framework using the Oculus Rift DK2 VR Head Mounted Display (HMD) and the Leap Motion hand tracking device. The Leap Motion was mounted on the front of the HMD facing the participants' hands, to facilitate hand interaction. Additionally, we used a Logitech Pro 1080p HD webcam positioned on top of the Oculus DK2, to record participants' hand from the user's perspective at all times. The system was developed using C\#, Unity 2018.2 and Leap Motion 4.0 SDK. The virtual interaction space was $60 \mathrm{~cm}$ x $60 \mathrm{~cm}$ x $60 \mathrm{~cm}$. The setup and equipment are shown in Fig 2.

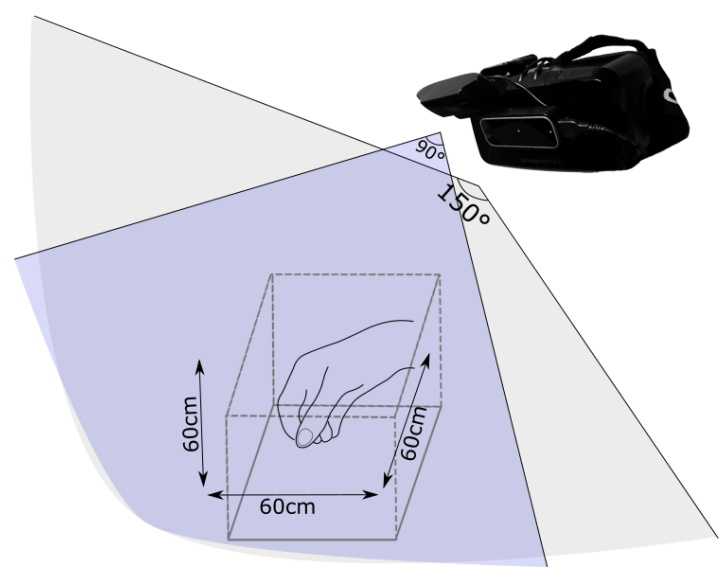

Figure 2. System configuration displaying the custom experimental framework: Leap Motion and Logitech Pro 1080p HD camera attached to the Oculus Rift DK2.

\section{Environment}

The experiment was conducted in a controlled environment under laboratory conditions at location $\mathrm{N} 52^{\circ} 28.35^{\prime} \mathrm{E} 53^{\circ}$ $5.87^{\prime}$ in July 2019. The average outdoor temperature was $22.4^{\circ} \mathrm{C}(\mathrm{SD} \mathrm{3.8})^{1}$ and the indoor controlled environment temperature was constant $20^{\circ} \mathrm{C}$ to minimise the potential inference of environmental and weather conditions in the results of the presented study. The test room was lit by a 2700k (warm white) fluorescent with no external light source.

The virtual environment showed a virtual desk with its surface aligned to a seating position, as in Figure 1(c). The virtual mug was placed at the centre of the table, changing its texture and content as presented in the next subsection and in Figures 1(d)-1(g). Additionally, the scenario showed a window, which changed views in between conditions.

\section{Conditions}

\section{Hand Representation}

Following Schwind et al. definitions [82], for hand representations we selected an abstract hand model that was extracted from the Leap Motion SDK and was represented as a set of cylinders and spheres representing bones and joints respectively (please refer to Figure 1(a). For the human hand condition we chose an androgynous model, following Schwind et al. recommendation for avoiding noticeable gender characteristics in human hands [82] (please refer to Figure 1(b)).

\section{Thermal Representations}

For this study, we chose a 3D mug as the interaction object, due to its familiarity in everyday tasks, as well as the variations of grasping it proposes. Won et al. [102] showed that the colour red is associated to hot concepts, blue to cold and green to reliable and safe. For the mug, we used the yellow colour, as it is the only one of the primary colours that was not associated to any connotation that may influence the study results.

\footnotetext{
${ }^{1}$ Weather data collected from: https://www . accuweather.com/en/ $\mathrm{gb} / \mathrm{birmingham} / \mathrm{b} 5-5 / j u l y$-weather/326966 (last accessed September 2019)
} 


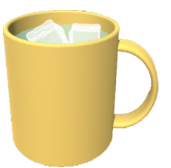

(a) Cold

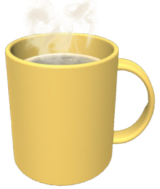

(b) Hot

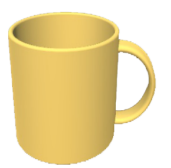

(c) Empty
Figure 3. Thermal representations for the virtual mug. 3(a) shows the cold condition with ice cubes and a clear liquid, 3(b) shows the hot condition with coffee steam coming out of the top and 3(c) shows the empty condition with no content.

- Cold: The mug content in this condition was a set of ice cubes inside a clear blue liquid (as in Figure 3(a)).

- Hot: The mug content was rendered as a brown liquid simulating coffee with steam coming from the top of the mug (as in Figure 3(b)).

- Empty: An empty mug with no content inside (as in Figure 3(c)).

We chose the colours of the liquid (blue for cold and brown for hot) based on previous literature showing that surfaces whose dominant frequencies are towards the blue end of the spectrum are perceived as cold, and those towards the red end of the spectrum are perceived as hot [8].

\section{Virtual Environment}

The environment surrounding objects has shown to influence interaction choice [99], therefore, to evaluate this in the context of the study, we explore different contextual representations to support the thermal cues above.

- Basic: Presents a simple yellow mug, in all 3 temperature conditions. As in Figure 1(d) the only visual difference between the thermal conditions is the rendered content inside the mug.

- Content Label: Presents a mug with a label attached to it, informing about the contents inside as in Figure 1(e).

- Glass: Presents a see-through mug in a transparent texture as in Figure 1(f). This allows the user to see the content of the mug through the mug itself, mimicking a glass texture.

- Context Objects: Presents the mug used in the basic condition accompanied by other contextual objects to support the thermal representation as in Figure 1(g). These accompanying objects were presented behind the mug, $10 \mathrm{~cm}$ away from its original position in both $\mathrm{z}$ and $\mathrm{x}$ axes. The accompanying items were a coffee espresso machine for the hot condition, ice bucket for the cold condition and an empty bucket for the empty condition. The view from the window also changed depending on the thermal cues, displaying a snowy landscape for the hot condition, a beach for the cold condition and a forest for the empty condition.

\section{Task}

Participants were instructed to pick and move the virtual mug from the origin location to a target location situated on the left of the original object and displayed as a 3D semi-transparent virtual mug in a different colour, as in Figure 4.

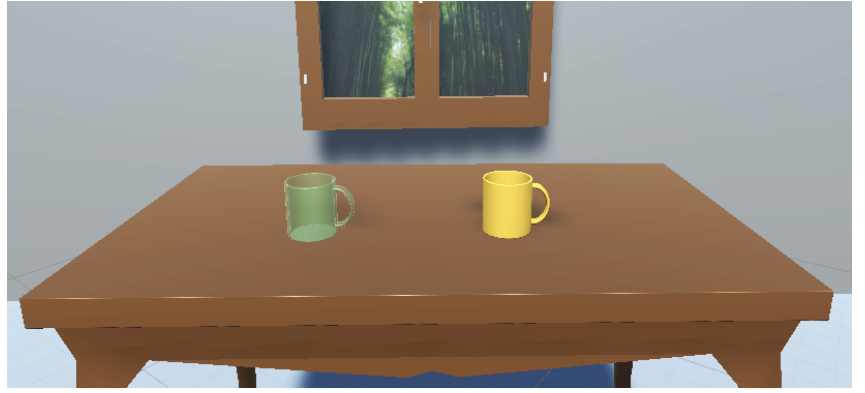

Figure 4. Interaction environment displaying the virtual mug (yellow virtual mug) and the target position (semi-transparent green virtual mug).

\section{Participants}

A total of 50 participants (21 females, 29 males) from a population of university students and staff members volunteered to take part in this study. Participants ranged in age from 18 to 50 $(\mathrm{M}=25.5, \mathrm{SD}=14.57)$. All participants were right-handed, to ensure they interacted with the mug under the same conditions (i.e. the handle in the same orientation with respect to their dominant hand).

All participants performed the experiment tasks under both hand conditions(abstract and human). Participants completed a standardised consent form and were not compensated. Visual acuity of participants was measured using a Snellen chart, each participant was also required to pass an Ishihara test to check for colour blindness. Participants with colour blindness and/or non corrected visual acuity of $<0.80$ (where 20/20 is 1.0) were not included in this study.

Participants were asked to self-assess their level of experience with VR systems, with 16 participants reporting to have an average level of experience, 31 reported being novice to the technology and 3 self-labelled themselves as experts. Participants did not have any previous experience with hand tracking sensors.

\section{Protocol}

Pre-test

Prior to the study, participants were given a written informed consent form, where the test protocol and main aim of the study were described. Additionally, participants completed a pre-test questionnaire enquiring about their background level of experience with VR systems and hand recognition sensors.

\section{Calibration}

Before each test, the test coordinator followed manufacturers' guidelines to help participants fit the HMD in the most suitable and comfortable way. The camera attached to the Oculus DK2 (Fig. 2) was adjusted each time to ensure the participants' hand was in the field of view of the devices.

\section{Training}

Participants were trained to pick and move a neutral object (a cube) from its original position to a target position, to familiarise themselves with the VR environment and hand interaction space. Thermal cues were not included at this stage of the study. Participants spent 7-10 minutes training with the system until they felt comfortable with the task and the apparatus. 
Test

Once participants were comfortable with the interaction space and the overall VR environment, they were presented with the main experimental task. Each participant completed 24 tasks(2 hand representations $\times 3$ temperature cues $\times 4$ environmental conditions as shown in Fig 1). The order of the hand representation conditions were counterbalanced; half participants started with human hand (Fig. 1(b)) and the other half with the abstract hand (Fig. 1(a)). Thermal and environmental conditions were then presented in randomised controlled order. Participants were asked to pick and move the virtual mug the way they felt most intuitive to them and instruct the test coordinator when they were happy with their hold. Hand tracking data was then recorded from the Leap Motion device, while an image was captured of both the VR scenario and from the Logitech webcam.

\section{Post-Test}

After each hand representation condition, participants were asked to complete the Igroup Presence Questionnaire (IPQ) and a set of tailored questions asking about their experience during interaction with the virtual object in different conditions.

\section{Metrics}

\section{Grasp Aperture}

We capture $X, Y, Z$ positions of finger joints during the interaction using the Leap Motion. We use the Grasp Aperture (GAp) defined in equation 1 from the grasp model presented in [3].

$$
G A p=\sqrt{\left(P_{x}-B_{x}\right)^{2}+\left(P_{y}-B_{y}\right)^{2}+\left(P_{z}-B_{z}\right)^{2}}
$$

Where $G A p$ is the distance between the index and the thumb fingers in the $\mathrm{x}, \mathrm{y}$ and $\mathrm{z}$ axes, and $P_{x}, P_{y}$ and $P_{z}$ are the coordinates of the index finger tip, and $B_{x}, B_{y}$ and $B_{z}$ are coordinates of the thumb tip.

\section{Labelling}

Two trained rater academics labelled all grasps individually; following the methodology described in [17]. The grasp types used for labelling are those in the Human GRASP taxonomy [28]. Additionally, raters labelled the grasp location of the mug as explained in section Grasp Location. The raters came from a computer science background and were familiar with human grasping literature. Raters were asked to label both the real view captured by the webcam and the virtual view captured in Unity when the object was grasped. The difference in the parameters between raters were analysed by rater 1 , who made a final decision about which rater's assignment was correct as in $[27,17]$.

\section{Grasp Type}

The full set of grasps used for labelling are those by Feix et al. in the Human GRASP taxonomy [28]. This taxonomy divides grasp types in three main categories: power, intermediate and precision. Power grasps are linked to stability and security and distinguished by large areas of contact between the hand and the object [21]. Precision grasps are commonly defined by the object being held between the finger tips, allowing an increased level of manipulation [53]. Intermediate grasps present elements of power and precision roughly in the same proportion, enabling a finer representation of grasp types [28].

\section{Grasp Location}

An object can be manipulated in different ways. For each way it is manipulated, there might be different proportions of the object relevant for the actual grasp. Therefore, Feix et. al. introduce the concept of grasped location, which they define as the local part of the object specific to the grasp instance (see Figure 5). An object can have multiple grasp locations, and humans will chose a grasp location based on the task and other parameters [26].

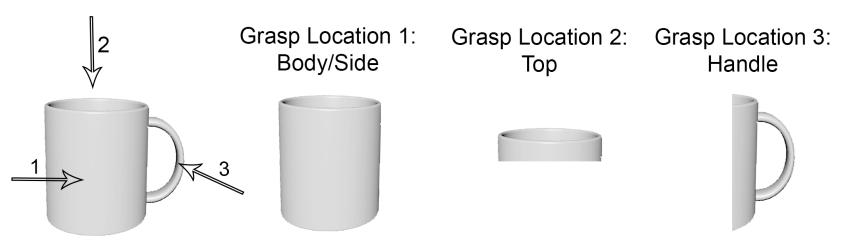

Figure 5. Grasp locations as by the Human Grasp Taxonomy [26] for the virtual mug used in this study.

\section{Presence Questionnaire}

The IPQ is a scale for measuring the sense of presence experienced in VR. When compared to other presence questionnaires, IPQ has shown to provide the highest reliability [81]. We used the IPQ to assess presence for each hand condition; human and abstract. The questionnaire is structured in 4 sub-scales: General Presence (PRES), Spatial Presence (SP), Involvement (INV) and Experience Realism (REAL), with 14 items in total, rated in 7-point scale (1-no feeling of presence, 7-strong feeling of presence). The scores for each sub-scale as well as the overall score are calculated by averaging their 7-point scores.

\section{Post-Test Questionnaire}

The post-test questionnaire consisted of tailored questions asking about the perceived usefulness of thermal visual cues. We used the post-test questionnaire, interview and observation to assess the perceived usefulness of visual cues for each hand condition.

\section{Hypotheses}

Following the current literature defined in this paper we propose the hypotheses listed below:

- $\mathbf{H}_{1}$ :The thermal visual cues of the object have an effect on the grasp metrics (i.e. aperture, location and type).

- $\mathbf{H}_{2}$ :The visual representation of the hand have a effect on the grasp metrics.

\section{Statistical Analysis}

The Shapiro-Wilk [85] normality test found the data to be not normally distributed. We test for significance between the conditions and the metrics described using a non parametric Friedman test [31]. 95\% Confidence Intervals (CI) and pair-wise Effect Sizes (ES) are reported following a non dichotomous statistical approach [24]. 


\section{RESULTS}

\section{Grasp Aperture (GAp)}

A comprehensive analysis of grasp aperture with Effect Sizes (ES) and 95\% Confidence Intervals (CIs) per temperature representation and scenario conditions is presented in Table 1 for the abstract hand and in Table 2 for the human hand.

\section{Hand Representations}

No statistically significant differences were found between the abstract and human hand visualisations for any of the environment conditions under study when comparing them pairwise by thermal cue for all the available scenarios (i.e. Hot abstract vs. hot human, empty abstract vs. empty human and cold abstract vs. cold human).

\section{Environment Conditions}

- Basic: Statistically significant differences were found between temperature representations for the abstract hand, with medium ES shown between the empty and hot conditions and between the cold and hot conditions (Table 1). Statistically significant differences were also found in the human hand condition, with large ES between the empty and hot conditions and medium ES between the cold and hot conditions, as shown in Table 2.

- Content Label: Both hand conditions presented statistically significant differences between temperature representations, with large ES shown between the empty and hot conditions (Tables 1 and 2). The human hand condition showed a medium ES between cold and hot conditions.

- Glass: Only the human hand presented statistically significant differences between the temperature representations, presenting large ES between the empty and hot and between the hot and cold conditions (Table 2).

- Context Objects: Statistically significant differences were presented between temperature representations in the $h u$ man hand condition with large ES between the empty and hot conditions and medium ES between the cold and hot conditions (Table 2).

Overall grasp aperture showed statistically significant differences for visual thermal cues in every condition under study with the human hand interaction while it only presented statistically significant differences in basic and content label conditions for the abstract hand model.

\section{Grasp Location}

Grasp location was defined as the preferred location for the grasp as in Figure 5. A comprehensive analysis of grasp location differences between visual thermal cues for all environment conditions is presented in Table 1 for the abstract hand and in Table 2 for the human hand. These tables present the statistical differences, Effect Sizes (ES) and visual heat-maps for the grasp location. Heat-maps are calculated to show the mid point between the thumb, and index finger during grasp.

\section{Hand Representations}

As with the grasp aperture no statistically significant differences were found between the abstract and the human hand visualisations for any of the context conditions under study when comparing them pairwise by temperature for all the available scenarios (i.e. Hot abstract vs. hot human, empty abstract vs. empty human and cold Abstract vs. cold human).

\section{Environment Conditions}

- Basic: Statistically significant differences were found between temperature representations for the abstract hand, with medium ES shown between the empty and hot conditions and between the cold and hot conditions (Table 1). Statistically significant differences were also found in the human hand condition, with large ES between the empty and hot conditions and between the cold and hot conditions (Table2).

- Content Label: Both hand conditions presented statistically significant differences between temperature representations, with large ES showing between the empty and hot conditions and medium ES between the cold and hot conditions as in Tables 1 and 2.

- Glass: Both hands presented statistically significant differences between the temperature representations, with medium ES between the empty and hot and between the hot and cold conditions (Tables 1 and 2).

- Context Objects: Statistically significant differences were presented for both hands with medium ES between empty and hot conditions (Tables 1 and 2).

Overall, statistically significant differences were presented for both hands across all environmental conditions with larger effect sizes between the empty and hot conditions and cold and hot conditions for both the abstract and the human hand. The results suggest a change in the location of the grasp for the hot content condition across all environmental conditions, which is consistent with the visual density representation in the heat-maps, where the hot conditions present a higher density of grasps around the handle area.

\section{Grasp Types}

In this section we report on grasp choice patterns for each grasped location (see Figure 5). Figure 6 presents grasp choices based on hand conditi on and mug content representation. A total of 1200 instances (50 participants $\times 2$ hand representations $\times 4$ environmental conditions $\times 3$ visual thermal cues) were labelled according to the methodology presented in the Labelling section. Each hand representation received 600 instances that were further analysed in the following paragraphs based on the grasp locations presented in Figure 5.

- Grasp Location 1 (Handle): $53.16 \%$ (355 instances) of the abstract hand data and 64\% (380 instances) of the human hand data were located in the handle of the mug.

- Abstract hand: Out of the 355 instances, $43.38 \%$ (154 instances, see Figure 6) of them belonged to hot content conditions while $31.54 \%$ (112 instances) were for cold content and the remaining $25.07 \%$ (89 instances) for empty conditions. 
Table 1. Abstract Hand - Grasp aperture and grasp location statistics, displaying effect sizes (IES|) and 95\% confidence intervals (CI) where H stands for Hot content, $C$ for Cold content and $E$ for Empty mug conditions.

\begin{tabular}{|c|c|c|c|c|c|}
\hline & & Basic & Content Label & Glass & Context Objects \\
\hline \multirow{3}{*}{ 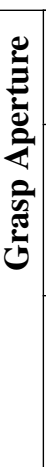 } & $p$ & $\begin{array}{c}(\text { Stat }=\mathbf{8 . 6 8} \\
\mathbf{p}=\mathbf{0 . 0 1})^{*}\end{array}$ & $\begin{array}{c}(\text { Stat }=\mathbf{1 3 . 0} \\
\mathbf{p}=\mathbf{0 . 0 2})^{*}\end{array}$ & $\begin{array}{c}(\text { Stat }=1.74 \\
p=0.06)\end{array}$ & $\begin{array}{c}(\text { Stat }=1.48 \\
p=0.47)\end{array}$ \\
\hline & $|\mathrm{ES}|$ & $\begin{array}{l}\mathbf{E} v s . \mathbf{H}=0.67 \\
\mathbf{E} v s . \mathbf{C}=0.13 \\
\mathbf{C} v s . \mathbf{H}=0.50\end{array}$ & $\begin{array}{l}\mathbf{E v s .} \mathbf{H}=0.86 \\
\mathbf{E} v s . \mathbf{C}=0.38 \\
\mathbf{C} v s . \mathbf{H}=0.44\end{array}$ & $\begin{array}{l}\mathbf{E} v s . \mathbf{H}=0.50 \\
\mathbf{E} v s . \mathbf{C}=0.22 \\
\mathbf{C} v s . \mathbf{H}=0.28\end{array}$ & $\begin{array}{l}\mathbf{E} v s . \mathbf{H}=0.46 \\
\mathbf{E} v s . \mathbf{C}=0.08 \\
\mathbf{C} v s . \mathbf{H}=0.38\end{array}$ \\
\hline & $\begin{array}{l}95 \% \\
\text { CI }\end{array}$ & 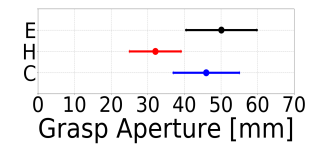 & 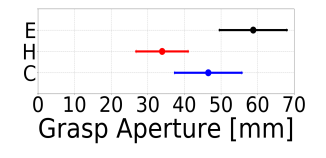 & 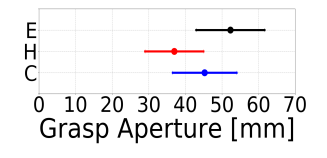 & 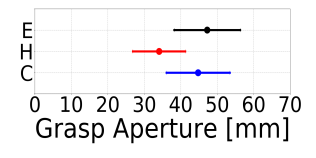 \\
\hline \multirow{3}{*}{ 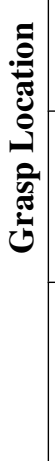 } & $p$ & $\begin{array}{c}(\mathbf{S t a t}=\mathbf{1 7 . 8 6} \\
\mathbf{p}<\mathbf{0 . 0 1})^{*}\end{array}$ & $\begin{array}{c}(\text { Stat }=\mathbf{2 3 . 5 1} \\
\mathbf{p}<\mathbf{0 . 0 1})^{*}\end{array}$ & $\begin{array}{c}(\text { Stat }=\mathbf{1 3 . 0 3} \\
\mathbf{p}=\mathbf{0 . 0 1})^{*}\end{array}$ & $\begin{array}{c}(\text { Stat }=\mathbf{8 . 2 2} \\
\mathbf{p}=\mathbf{0 . 0 2})^{*}\end{array}$ \\
\hline & $|\mathrm{ES}|$ & $\begin{array}{l}\mathbf{E v s .} \mathbf{H}=0.72 \\
\mathbf{E} v s . \mathbf{C}=0.17 \\
\mathbf{C} v s . \mathbf{H}=0.54\end{array}$ & $\begin{array}{l}\mathbf{E} v s . \mathbf{H}=0.84 \\
\mathbf{E} v s . \mathbf{C}=0.29 \\
\mathbf{C} v s . \mathbf{H}=0.48\end{array}$ & $\begin{array}{l}\mathbf{E} v s . \mathbf{H}=0.65 \\
\mathbf{E} v s . \mathbf{C}=0.23 \\
\mathbf{C} v s . \mathbf{H}=0.40\end{array}$ & $\begin{array}{l}\mathbf{E} v s . \mathbf{H}=0.52 \\
\mathbf{E} v s . \mathbf{C}=0.06 \\
\mathbf{C} v s . \mathbf{H}=0.45\end{array}$ \\
\hline & $\begin{array}{l}\text { Heat- } \\
\text { maps }\end{array}$ & 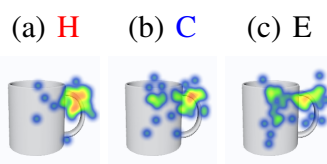 & $\begin{array}{lll}\text { (d) } \mathrm{H} & \text { (e) } \mathrm{C} & \text { (f) } \mathrm{E} \\
\therefore & \end{array}$ & $\begin{array}{lll}\text { (g) } \mathrm{H} & \text { (h) } \mathrm{C} & \text { (i) } \mathrm{E} \\
: 2 \beta & \text { : }\end{array}$ & 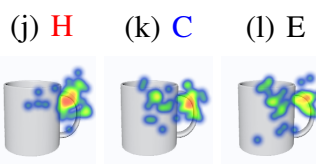 \\
\hline
\end{tabular}

Table 2. Human Hand - Grasp aperture and grasp location statistics, displaying effect sizes (|ES|) and 95\% confidence intervals (CI) where H stands for Hot content, $\mathrm{C}$ for Cold content and $\mathrm{E}$ for empty mug conditions.

\begin{tabular}{|c|c|c|c|c|c|}
\hline & & Basic & Content Label & Glass & Context Objects \\
\hline \multirow{3}{*}{ 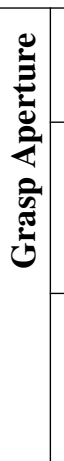 } & $p$ & $\begin{array}{c}(\text { Stat }=\mathbf{2 6 . 2 8} \\
\mathbf{p}<0.01)^{*}\end{array}$ & $\begin{array}{c}(\text { Stat }=22.84 \\
\mathbf{p}<\mathbf{0 . 0 1})^{*}\end{array}$ & $\begin{array}{l}(\text { Stat }=7.8 \\
\mathbf{p}=0.02)^{*}\end{array}$ & $\begin{array}{c}(\text { Stat }=\text { 11.68 } \\
\mathbf{p}=\mathbf{0 . 0 3})^{*}\end{array}$ \\
\hline & $|\mathrm{ES}|$ & $\begin{array}{l}\mathbf{E} v s . \mathbf{H}=0.95 \\
\mathbf{E} v s . \mathbf{C}=0.27 \\
\mathbf{C} v s . \mathbf{H}=0.65\end{array}$ & $\begin{array}{l}\mathbf{E} v s . \mathbf{H}=0.96 \\
\mathbf{E} v s . \mathbf{C}=0.35 \\
\mathbf{C} v s . \mathbf{H}=0.58\end{array}$ & $\begin{array}{l}\mathbf{E} v s . \mathbf{H}=0.82 \\
\mathbf{E} v s . \mathbf{C}=0.05 \\
\mathbf{C} v s . \mathbf{H}=0.79\end{array}$ & $\begin{array}{l}\mathbf{E} v s . \mathbf{H}=0.90 \\
\mathbf{E} v s . \mathbf{C}=0.38 \\
\mathbf{C} v s . \mathbf{H}=0.46\end{array}$ \\
\hline & $\begin{array}{c}95 \% \\
\mathrm{CI}\end{array}$ & 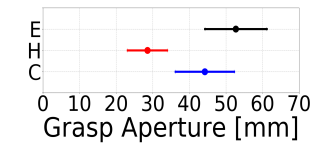 & 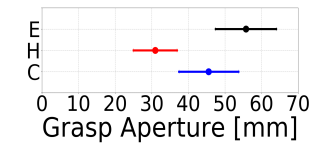 & 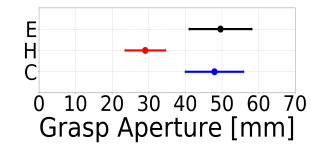 & 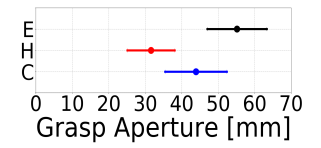 \\
\hline \multirow{3}{*}{ 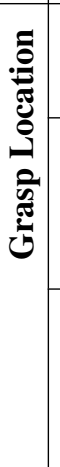 } & $p$ & $\begin{array}{c}(\text { Stat }=\mathbf{2 7 . 1 8} \\
\mathbf{p}<\mathbf{0 . 0 1})^{*}\end{array}$ & $\begin{array}{c}(\text { Stat }=\mathbf{2 6 . 6 9} \\
\mathbf{p}<\mathbf{0 . 0 1})^{*}\end{array}$ & $\begin{array}{c}(\text { Stat }=21.5 \\
\mathbf{p}=\mathbf{0 . 0 2})^{*}\end{array}$ & $\begin{array}{c}(\text { Stat }=\text { 13.67 } \\
\mathbf{p}<\mathbf{0 . 0 1})^{*}\end{array}$ \\
\hline & $|\mathrm{ES}|$ & $\begin{array}{l}\mathbf{E} v s . \mathbf{H}=0.98 \\
\mathbf{E} v s . \mathbf{C}=0.14 \\
\mathbf{C} v s . \mathbf{H}=0.79\end{array}$ & $\begin{array}{l}\mathbf{E} v s . \mathbf{H}=0.95 \\
\mathbf{E} v s . \mathbf{C}=0.38 \\
\mathbf{C} v s . \mathbf{H}=0.60\end{array}$ & $\begin{array}{l}\mathbf{E} v s . \mathbf{H}=0.75 \\
\mathbf{E} v s . \mathbf{C}=0.05 \\
\mathbf{C} v s . \mathbf{H}=0.71\end{array}$ & $\begin{array}{l}\mathbf{E} v s . \mathbf{H}=0.62 \\
\mathbf{E} v s . \mathbf{C}=0.19 \\
\mathbf{C} v s . \mathbf{H}=0.40\end{array}$ \\
\hline & $\begin{array}{l}\text { Heat- } \\
\text { maps }\end{array}$ & $\begin{array}{lll}\text { (a) } \mathrm{H} & \text { (b) } \mathrm{C} & \text { (c) } \mathrm{E} \\
& \end{array}$ & 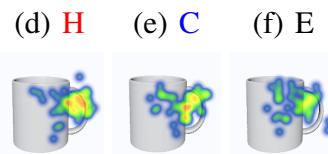 & 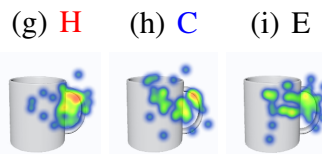 & 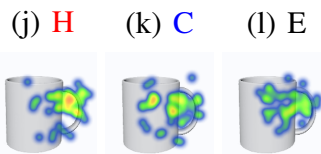 \\
\hline
\end{tabular}




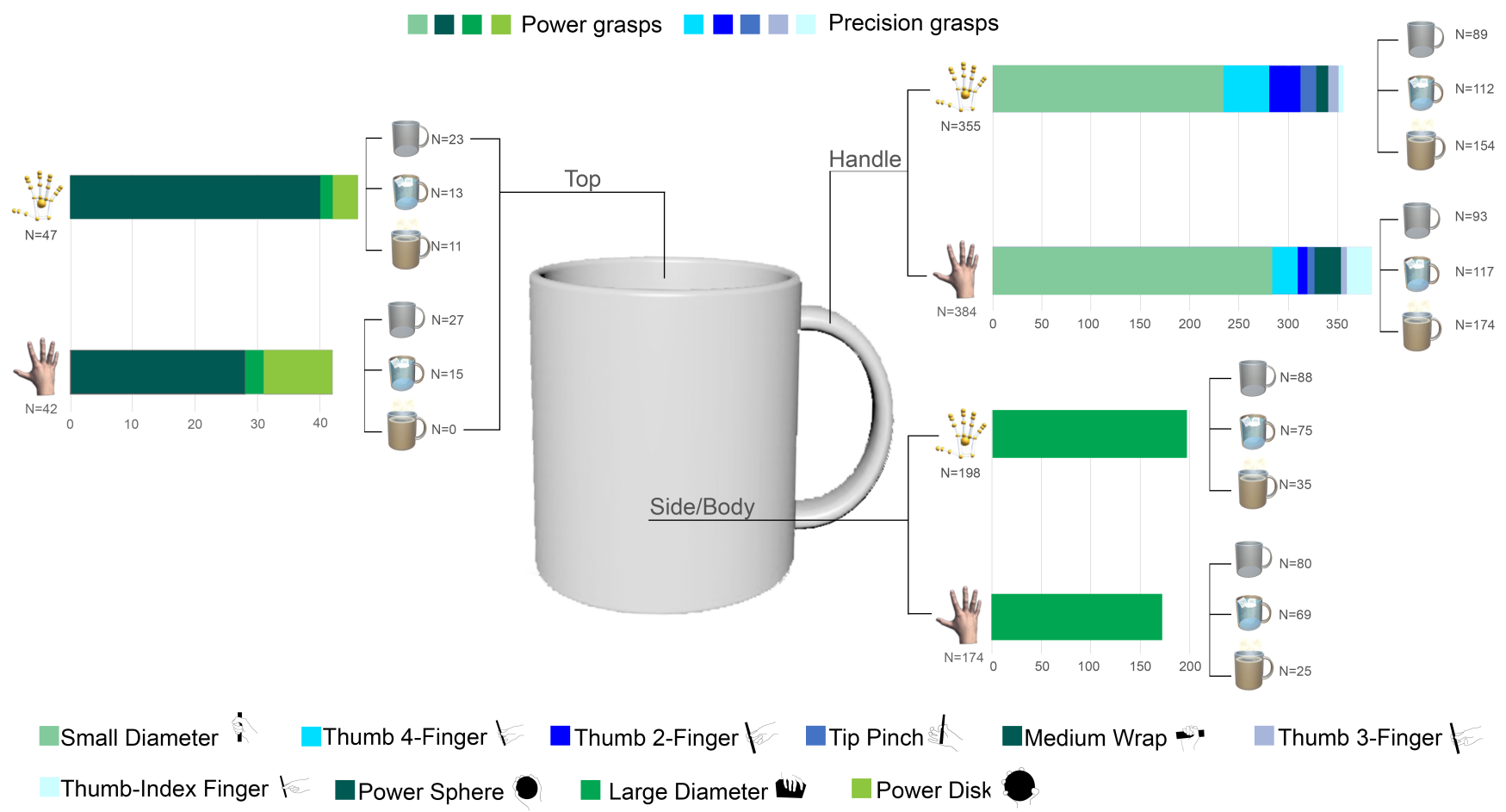

Figure 6. Grasp type choice for each grasped location; N represents the number of instances for which that grasp location was chosen, for each temperature condition. Grasp types are categorised in Power (variations of green) and Precision (variations of blue).

- Human hand: Handle grasps for the human hand were divided as follows: $45.31 \%$ (174 instances as in Figure 6) for the hot conditions, $30.46 \%$ (117 instances) for the cold conditions and the remaining $24.22 \%$ (93 instances) for the empty conditions.

The main grasp choice for this location was the Small Diameter grasp for both hand conditions. This grasp belongs to the Power Grasp category defined in the Grasp Types section, thus a stable grasp enabling the firm grip of the virtual mug. The remaining grasps belonged to the Precision Grasp category, enabling a precise grasp of the mug using the fingertips. All grasp choices identified in the dataset for the handle location are reported in detail in Figure 6.

- Grasp Location 2 (Top): A total of 47 instances (7.83\% of the dataset) for the abstract hand and 42 (7\% of the dataset) for the human hand conditions were located around the top of the mug.

- Abstract hand: Out of the 47 instances, $23.40 \%$ (11, see Figure 6) belonged to hot content conditions while $27.66 \%$ (13 instances) were for cold content and the remaining $48.94 \%$ (23 instances) for empty conditions.

- Human hand: $35.71 \%$ (15 instances) for the cold conditions and the remaining $64.28 \%$ (27 instances) for the empty conditions. No grasps were recorded in this area for the hot condition in the human hand visualisation.

All grasps recorded in the top of the mug for both hand conditions belonged to the Power Grasp category as defined in the Grasp Types section. Figure 6 displays grasp choice in more detail.

- Grasp Location 3 (Body/Side): 33\% (198 instances) of the abstract hand condition and 20\% (174 instances) of the human hand condition were grasps recorded in the body/side of the mug.

- Abstract hand: Out of the 198 instances, 17.67\% (35 as in Figure 6) of them belonged to hot content conditions while $37.88 \%$ (75 instances) were for cold content and the remaining $44.44 \%$ (88 instances) for empty conditions.

- Human hand: 14.36\% (25 instances as in Figure 6) for the hot conditions, 39.65\% (69 instances) for the cold conditions and the remaining $45.97 \%$ (80 instances) for the empty conditions.

The only grasp type recorded in this area was a Large Diameter grasp, from the Power Grasp category. This grasp is categorised by large areas of the hand in direct contact with the object, enabling a firm grip which is in alignment with the location of the grasp in the virtual mug.

\section{IPQ}

The IPQ Presence Questionnaire showed no statistically significant differences between the hand representations under study, with the abstract hand obtaining an average score of $(\mathrm{M}=4.54$, $\mathrm{SD}=1.02)$ and the human hand $(\mathrm{M}=4.75 \mathrm{SD}=1)$. None of the 
sub-scales of the questionnaire showed any statistically significant results. Presence scores by sub-scale are presented in Figure 7.

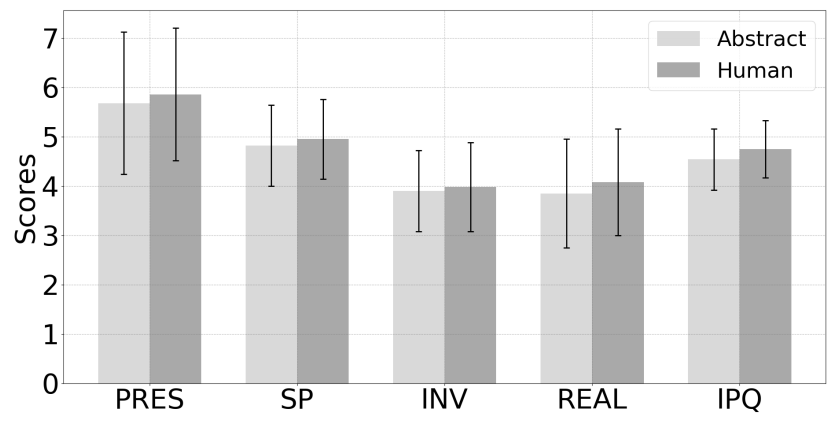

Figure 7. Scores for IPQ sub-scales and overall IPQ score for abstract and human hand conditions; a score equal to 7 represents the highest feeling of presence while 1 represents the lowest.

\section{Post-Test Questionnaire}

Participants were asked to complete a post-test questionnaire to gain a better understanding of their perceptions while interacting with the thermal cues, the environment and hand representations.

Participants reported ice and steam visual cues from the thermal representations section in Conditions as the strongest visual cue supporting their grasping behaviour. However, these are the cues they were more familiar with, as they were present in all environment conditions under study for both hand representations.

Following these remarks, participants were asked if they felt their grasp location and type was influenced by the different thermal representations or the environment. 37 participants (abstract hand) and 36 participants (human hand) reported that grasp location was influenced by mug content. Additionally, 29 participants (abstract hand) and 37 (human hand) reported that the visual thermal representation influenced the location where they grasped. Some participants reported "I used the handle because I did not want to get burnt or my hand to be too cold." [P34] or "I used the handle for hot content to avoid being burnt" [P28] while other participants reported "Not necessarily as I wasn't too concerned about burning my hand (because it is robotic [sic]), therefore, didn't matter how I grasped the mug " [P03].

Some additional comments included: "With real hand, I almost expected the mugs to have different weights with respect to the amount of liquid in each mug. This was to a greater extent than the abstract hand." [P11], "I felt that the simulation with the human hand made me feel the need to be careful in case I burnt myself a lot more than when using the robot hand simulation."[P24], "I first thought that it was my hand and I realised that it was not only by looking at the short nails."[P47].

Overall, participants reported that mug content and thermal cues have a stronger impact on their grasp choice and loca- tion than other environmental cues such as changes in the environment or in the hand representation.

\section{DISCUSSION}

We present a study to evaluate the influence of visual thermal cues on VR grasp type, aperture and location. Our results show how visual thermal feedback had an influence on grasp aperture $(G A p)$, grasp location and grasp type, thus we accept the hypothesis $\mathbf{H}_{\mathbf{1}}$ that thermal visual cues have an effect on grasp metrics. Additionally, our results correlate with prior work studying grasp patterns for real objects, therefore showing that visual thermal feedback has an effect on grasping approach [69] and indicating a connection between grasping approaches across both VR and real environments.

Our results shown that visual representations of temperature influenced $G A p$ for all conditions under study when using the human hand (Figure 1(b)) and for the Basic (Figure 1(d)) and Content Labels (Figure 1(e)) conditions when using the abstract hand. Participants grasped the virtual mug with a smaller GAp in the hot condition compared to cold and empty for both hand representations (abstract and human).

Grasping instances presenting a small $G A p$ were predominantly located around the handle of the virtual mug, showing the influence of the size of the grasped location on the grasp pattern when interacting with virtual objects. This correlates with grasping real objects, where the aperture of the grasp is primarily influenced by the size of the grasped location [26]. Participants predominantly used the handle as a grasp location in both hot and cold conditions, while grasp location choice varied between body/side, top or handle for the empty condition. This shows that the visual representation of content inside the mug influenced user's behaviour when grasping, and therefore could illustrate a strong functional correlation between the shape of the grasped location and the manner in which it is generally grasped by the hand which has been shown in real object grasping [76]. Similarly, our results highlighted that the object shape and the chosen grasped location had an influence on the grasp used. Participants predominantly chose a Large Diameter for body/side (cylinder shape) and a Power Sphere for top (disk shape) on the mug.

As with previous studies [66, 101], participants did not show an awareness of the number of fingers involved while interacting with the system. Commonly, users presented different variations of the thumb-finger grasp for the same grasp location; notably variations of Thumb-Index Finger, Thumb 2-Finger, Thumb 3-Finger, Thumb 4-Finger when grasping the handle. Differences in grasp type were identified between the human and abstract hand conditions; for instances where the handle was chosen, participants used a power grasp in $80.72 \%$ of the instances in the human hand condition, while choosing power grasps in only $69.29 \%$ of the instances in the abstract hand condition. This result suggests that users intuitively performed a grasp that could normally hold a heavy object more often with the human hand than with the abstract hand, with power grasps being associated with stability and security when grasping real objects[21]. 
When comparing between conditions, ES was generally smaller for the abstract hand than for the human hand, however, there were no significant statistical differences between hand representations for grasp data. The IPQ scores did not present statistically significant differences in perceived presence between hand representations. This finding is contrary to popular literature [83], and therefore we reject our hypothesis $\mathbf{H}_{\mathbf{2}}$ for the effect of hand representations on grasp metrics. However, during the interview and post-questionnaire within the study, participants emphasised higher levels of attachment to the human hand condition.

Throughout the study, participants were not specifically instructed that grasping type, location and aperture were under study and were therefore free to interact with the virtual mug as they felt suitable. However, they could have experienced the Good Subject Effect. Notably, this is found when participants can respond to an experiment in ways that they believe confirm the hypothesis of the study [57]. Although we believe our methodology negates this effect, it can not be ruled out completely. Therefore, future work should consider masking the temperature representations or embedding the "pick and move" task in a more complex task or environment and further assess current results. Additionally, future studies could integrate colour and context variations, building up the current knowledge in grasping presented in this evaluation.

Overall, we presented the first study into understanding the influence of visual thermal cues on grasping patterns, with practical implications for the VR interaction design community:

- Grasp aperture: Visual thermal feedback on an object had an influence on grasp aperture $(G A p)$ showing smaller apertures for visual representations of hot content. This insight should be considered for interaction virtual object design in VR environments where the user needs to manipulate thermally variable objects (i.e. welding training scenarios as in $[9,68])$.

- Grasp location and type: Objects representing a weight or content showcased grasp location differences when compared to the empty mug condition, with users presenting a higher density of precision grasps in the handle area. Participants performed more precise grasps for triggering the interaction when the mug had content, therefore suggesting that the perceived weight, content and/or fragility of an object had an effect on grasp choice and location. This interaction insight is useful for designers creating VR environments that require direct interaction with objects, specially for training (i.e. VR training environments in construction and manufacturing [6]). In these scenarios, the form and shape of objects need to be considered, with objects being designed to specifically encourage this user selection of precision grasps and thus facilitating a more precise and finer manipulation.

\section{CONCLUSION}

The presented study evaluates the influence of visual thermal cues in VR on grasping. We report on a within participants study to evaluate how thermal cues (empty hot and cold) affect user grasp metrics, notably grasp location, grasp aperture $(G A p)$ and grasp choice. Our results illustrate that visual thermal feedback in VR can have an influence on user grasp location when aiming to pick up and translate objects of different content (i.e a mug with hot coffee or cold water). Additionally, we report on the influence on grasp metrics such as grasp aperture and grasp type and show that higher effect sizes can be observed between the hot-cold and hot-empty conditions. While future work could analyse other virtual objects or thermal cues, our findings present the first study into understanding the influence that visual cues have on user grasping in VR.

\section{REFERENCES}

[1] Merwan Achibet, Adrien Girard, Anthony Talvas, Maud Marchal, and Anatole Lećuyer. 2015. Elastic-Arm: Human-scale passive haptic feedback for augmenting interaction and perception in virtual environments. In 2015 IEEE Virtual Reality (VR). 63-68. DOI: http://dx.doi.org/10.1109/VR.2015.7223325

[2] Vamsi Kiran Adhikarla, Pawel Wozńiak, Attila Barsi, Dave Singhal, Petér T. Kovaćs, and Tibor Balogh. 2014. Freehand interaction with large-scale 3D map data. In 2014 3DTV-Conference: The True Vision - Capture, Transmission and Display of $3 D$ Video (3DTV-CON). 1-4. DOI: http://dx.doi.org/10.1109/3DTV.2014.6874711

[3] Maadh Al-Kalbani, Ian Williams, and Maite Frutos-Pascual. 2016a. Analysis of Medium Wrap Freehand Virtual Object Grasping in Exocentric Mixed Reality. In Mixed and Augmented Reality (ISMAR), 2016 IEEE International Symposium on. IEEE, 84-93.

[4] Maadh Al-Kalbani, Ian Williams, and Maite Frutos-Pascual. 2016b. Improving freehand placement for grasping virtual objects via dual view visual feedback in mixed reality. In Proceedings of the 22nd ACM Conference on Virtual Reality Software and Technology. ACM, 279-282.

[5] Bruno Araujo, Ricardo Jota, Varun Perumal, Jia Xian Yao, Karan Singh, and Daniel Wigdor. 2016. Snake Charmer: Physically Enabling Virtual Objects. In Proceedings of the TEI'16: Tenth International Conference on Tangible, Embedded, and Embodied Interaction. ACM, 218-226.

[6] R Barkokebas, C Ritter, V Sirbu, X Li, and M Al-Hussein. 2019. Application of Virtual Reality in Task Training in the Construction Manufacturing Industry. In ISARC. Proceedings of the International Symposium on Automation and Robotics in Construction, Vol. 36. IAARC Publications, 796-803.

[7] Hrvoje Benko, Christian Holz, Mike Sinclair, and Eyal Ofek. 2016. NormalTouch and TextureTouch: High-fidelity 3D Haptic Shape Rendering on Handheld Virtual Reality Controllers. In Proceedings of the 29th Annual Symposium on User Interface Software and Technology (UIST '16). ACM, 717-728. DOI : http://dx.doi.org/10.1145/2984511.2984526 
[8] Corwin A. Bennett and Paule Rey. 1972. What's So Hot about Red? Human Factors 14, 2 (1972), 149-154. DOI : http://dx.doi.org/10.1177/001872087201400204 PMID: 5022474.

[9] VG Bharath and Rajashekar Patil. 2018. Solid modelling interaction with sensors for virtual reality welding. In MATEC Web of Conferences, Vol. 144. EDP Sciences, 01008 .

[10] Frank Biocca. 1995. Communication Applications of Virtual Reality. (06 1995).

[11] Frank Biocca, Jin Kim, and Yung Choi. 2001. Visual Touch in Virtual Environments: An Exploratory Study of Presence, Multimodal Interfaces, and Cross-Modal Sensory Illusions. Presence: Teleoperators and Virtual Environments 10, 3 (2001), 247-265. DOI: http://dx.doi.org/10.1162/105474601300343595

[12] Christoph W Borst and Arun P Indugula. 2005. Realistic virtual grasping. In IEEE Proceedings. VR 2005. Virtual Reality, 2005. 91-98.

[13] Christoph W Borst and Arun P Indugula. 2006. A spring model for whole-hand virtual grasping. Presence: Teleoperators and Virtual Environments 15, 1 (2006), 47-61.

[14] Doug A. Bowman, Ryan P. McMahan, and Eric D. Ragan. 2012. Questioning Naturalism in 3D User Interfaces. Commun. ACM 55, 9 (Sept. 2012), 78-88. DOI : http://dx.doi .org/10.1145/2330667.2330687

[15] Volkert Buchmann, Trond Nilsen, and Mark Billinghurst. 2005. Interaction with Partially Transparent Hands and Objects. In Proceedings of the Sixth Australasian Conference on User Interface - Volume 40 (AUIC '05). 17-20.

http://dl .acm.org/citation. $\mathrm{cfm} ? \mathrm{id}=1082243.1082246$

[16] Ian M. Bullock, Thomas Feix, and Aaron M. Dollar. 2013. Finding small, versatile sets of human grasps to span common objects. In 2013 IEEE International Conference on Robotics and Automation. 1068-1075. DOI : http://dx.doi.org/10.1109/ICRA.2013.6630705

[17] Ian M Bullock, Thomas Feix, and Aaron M Dollar. 2015. The Yale human grasping dataset: Grasp, object, and task data in household and machine shop environments. The International Journal of Robotics Research 34, 3 (2015), 251-255.

[18] Inrak Choi, Eyal Ofek, Hrvoje Benko, Mike Sinclair, and Christian Holz. 2018. CLAW: A Multifunctional Handheld Haptic Controller for Grasping, Touching, and Triggering in Virtual Reality. In Proceedings of the 2018 CHI Conference on Human Factors in Computing Systems (CHI'18). Article 654, 13 pages. DOI : http://dx.doi.org/10.1145/3173574.3174228

[19] Natalia Cooper, Ferdinando Milella, Carlo Pinto, Iain Cant, Mark White, and Georg Meyer. 2018. The effects of substitute multisensory feedback on task performance and the sense of presence in a virtual reality environment. PloS one 13, 2 (2018), e0191846.
[20] James J. Cummings and Jeremy N. Bailenson. 2016. How Immersive Is Enough? A Meta-Analysis of the Effect of Immersive Technology on User Presence. Media Psychology 19, 2 (2016), 272-309. DOI : http://dx.doi.org/10.1080/15213269.2015.1015740

[21] Mark R. Cutkosky. 1989. On grasp choice, grasp models, and the design of hands for manufacturing tasks. IEEE Transactions on Robotics and Automation 5, 3 (Jun 1989), 269-279. DOI : http://dx.doi.org/10.1109/70.34763

[22] Dragos Datcu and Stephan Lukosch. 2013. Free-hands Interaction in Augmented Reality. In Proceedings of the 1st Symposium on Spatial User Interaction (SUI'13). ACM, 33-40. DOI : http://dx.doi.org/10.1145/2491367.2491370

[23] Huong Q. Dinh, Neff Walker, Larry F. Hodges, Chang Song, and Akira Kobayashi. 1999. Evaluating the importance of multi-sensory input on memory and the sense of presence in virtual environments. In Proceedings IEEE Virtual Reality (Cat. No. 99CB36316). 222-228. D0I : http://dx.doi.org/10.1109/VR. 1999.756955

[24] Pierre Dragicevic. 2016. Fair statistical communication in HCI. In Modern Statistical Methods for HCI. Springer, 291-330.

[25] T. Feix, I. M. Bullock, and A. M. Dollar. 2014a. Analysis of Human Grasping Behavior: Object Characteristics and Grasp Type. IEEE Transactions on Haptics 7, 3 (July 2014), 311-323. DOI : http://dx.doi.org/10.1109/TOH. 2014.2326871

[26] Thomas Feix, Ian M. Bullock, and Aaron M. Dollar. 2014b. Analysis of Human Grasping Behavior: Object Characteristics and Grasp Type. IEEE Transactions on Haptics 7, 3 (July 2014), 311-323. DOI : http://dx.doi.org/10.1109/TOH. 2014.2326871

[27] Thomas Feix, Roland Pawlik, Heinz-Bodo Schmiedmayer, Javier Romero, and Danica Kragiç. 2009. A comprehensive grasp taxonomy. Robotics, Science and Systems Conference: Workshop on Understanding the Human Hand for Advancing Robotic Manipulation (2009), 2-3.

[28] Thomas Feix, Javier Romero, Heinz-Bodo Schmiedmayer, Aaron M. Dollar, and Danica Kragiç. 2016. The GRASP Taxonomy of Human Grasp Types. IEEE Transactions on Human-Machine Systems 46, 1 (Feb 2016), 66-77. DOI : http://dx.doi .org/10.1109/THMS . 2015.2470657

[29] Scott Fisher, M McGreevy, J Humphries, and W Robinett. 1987. Virtual environment display system. Proceedings of the Workshop on Interactive 3-D Graphics 1 (01 1987), 77-87. DOI : http://dx.doi.org/10.1145/319120.319127

[30] J. Randall Flanagan and Roland S. Johansson. 2001. Hand Movements. 
[31] Milton Friedman. 1940. A comparison of alternative tests of significance for the problem of $\mathrm{m}$ rankings. The Annals of Mathematical Statistics 11, 1 (1940), 86-92.

[32] Julia Fröhlich and Ipke Wachsmuth. 2013. The Visual, the Auditory and the Haptic âĂŞ A User Study on Combining Modalities in Virtual Worlds, Vol. 8021. 159-168. DOI:

http://dx.doi.org/10.1007/978-3-642-39405-8_19

[33] Daniel Harley, Alexander Verni, Mackenzie Willis, Ashley Ng, Lucas Bozzo, and Ali Mazalek. 2018. Sensory VR: Smelling, Touching, and Eating Virtual Reality. In Proceedings of the Twelfth International Conference on Tangible, Embedded, and Embodied Interaction (TEI'18). ACM, New York, NY, USA, 386-397. DOI :

http://dx.doi.org/10.1145/3173225.3173241

[34] Otmar Hilliges, David Kim, Shahram Izadi, Malte Weiss, and Andrew Wilson. 2012. HoloDesk: direct 3d interactions with a situated see-through display. In Proceedings of the SIGCHI Conference on Human Factors in Computing Systems. ACM, 2421-2430.

[35] Daniel Holz, Sebastian Ullrich, Marc Wolter, and Torsten Kuhlen. 2008. Multi-Contact Grasp Interaction for Virtual Environments. JVRB - Journal of Virtual Reality and Broadcasting 5(2008), 7 (2008).

[36] Sarah Hudson, Sheila Matson-Barkat, Nico Pallamin, and Guillaume Jegou. 2018. With or without you? Interaction and immersion in a virtual reality experience. Journal of Business Research (2018). DOI: http://dx.doi.org/https: //doi.org/10.1016/j.jbusres.2018.10.062

[37] Jacek Jankowski and Martin Hachet. 2015. Advances in interaction with 3D environments. In Computer Graphics Forum, Vol. 34. Wiley Online Library, 152-190.

[38] Yukun Jia and Abraham G. Campbell. 2017. Immersive Virtual Reality Training Tool for IoT Device Placement. In Proceedings of the 10th EAI International Conference on Simulation Tools and Techniques (SIMUTOOLS '17). 81-86. DOI : http://dx.doi .org/10.1145/3173519.3173521

[39] Noriko Kamakura, Michiko Matsuo, Harumi Ishii, Fumiko Mitsuboshi, and Yoriko Miura. 1980. Patterns of Static Prehension in Normal Hands. 34 (08 1980), $437-45$.

[40] Konstantina Kilteni, Raphaela Groten, and Mel Slater. 2012. The Sense of Embodiment in Virtual Reality. Presence Teleoperators amp Virtual Environments 21 (11 2012). DOI : http://dx.doi.org/10.1162/PRES_a_00124

[41] David Kim, Otmar Hilliges, Shahram Izadi, Alex D. Butler, Jiawen Chen, Iason Oikonomidis, and Patrick Olivier. 2012. Digits: Freehand 3D Interactions Anywhere Using a Wrist-worn Gloveless Sensor. In
Proceedings of the 25th Annual ACM Symposium on User Interface Software and Technology (UIST'12). ACM, New York, NY, USA, 167-176. DOI : http://dx.doi.org/10.1145/2380116.2380139

[42] Pascal Knierim, Valentin Schwind, Anna Maria Feit, Florian Nieuwenhuizen, and Niels Henze. 2018. Physical Keyboards in Virtual Reality: Analysis of Typing Performance and Effects of Avatar Hands. In Proceedings of the 2018 CHI Conference on Human Factors in Computing Systems (CHI'18). ACM, New York, NY, USA, Article 345, 9 pages. DOI : http://dx.doi.org/10.1145/3173574.3173919

[43] PrzemysÅĆaw Krompiec and Kyoungju Park. 2019. Enhanced Player Interaction Using Motion Controllers for First-Person Shooting Games in Virtual Reality. IEEE Access 7 (2019), 124548-124557. DOI : http://dx.doi.org/10.1109/ACCESS.2019.2937937

[44] Piyush Kumar, Jyoti Verma, and Shitala Prasad. 2012. Hand data glove: A wearable real-time device for human-computer interaction. 43 (01 2012), 15-26.

[45] Glyn Lawson, Davide Salanitri, and Brian Waterfield. 2016. Future directions for the development of virtual reality within an automotive manufacturer. Applied Ergonomics 53 (2016), 323 - 330. DOI:

http://dx.doi.org/https: //doi.org/10.1016/j.apergo.2015.06.024 Transport in the 21st Century: The Application of Human Factors to Future User Needs.

[46] Susan J Lederman and Roberta L Klatzky. 1987. Hand movements: A window into haptic object recognition. Cognitive Psychology 19, 3 (1987), 342 - 368. DOI: http://dx.doi.org/https: //doi.org/10.1016/0010-0285(87)90008-9

[47] Jaeyeon Lee, Michael Sinclair, Mar Gonzalez-Franco, Eyal Ofek, and Christian Holz. 2019. TORC: A Virtual Reality Controller for In-Hand High-Dexterity Finger Interaction. 1-13. DOI : http://dx.doi.org/10.1145/3290605.3300301

[48] Lorraine Lin and Sophie Jörg. 2016. Need a Hand?: How Appearance Affects the Virtual Hand Illusion. In Proceedings of the ACM Symposium on Applied Perception (SAP '16). 69-76. DOI : http://dx.doi.org/10.1145/2931002.2931006

[49] Sikun Lin, Hao Fei Cheng, Weikai Li, Zhanpeng Huang, Pan Hui, and Christoph Peylo. 2017. Ubii: Physical World Interaction Through Augmented Reality. IEEE Transactions on Mobile Computing 16, 3 (2017), 872-885.

[50] Axel Loewe, Emanuel Poremba, Tobias G. Oesterlein, Nicolas Pilia, Micha Pfeiffer, Olaf Doessel, and Stefanie Speidel. 2017. An interactive virtual reality environment for analysis of clinical atrial arrhythmias and ablation planning. In 2017 Computing in Cardiology (CinC). 1-4. DOI : http://dx.doi .org/10.22489/CinC.2017.125-118 
[51] Pedro Lopes, Sijing You, Lung-Pan Cheng, Sebastian Marwecki, and Patrick Baudisch. 2017. Providing Haptics to Walls \&\#38; Heavy Objects in Virtual Reality by Means of Electrical Muscle Stimulation. In Proceedings of the 2017 CHI Conference on Human Factors in Computing Systems (CHI '17). ACM, New York, NY, USA, 1471-1482. DOI :

http://dx.doi.org/10.1145/3025453.3025600

[52] Jean-Luc Lugrin, Johanna Latt, and Marc Latoschik. 2015. Avatar Anthropomorphism and Illusion of Body Ownership in VR. DOI :

http://dx.doi.org/10.1109/VR.2015.7223379

[53] Damian Lyons. 1985. A simple set of grasps for a dextrous hand. In Robotics and Automation. Proceedings. 1985 IEEE International Conference, Vol. 2. $588-593$.

[54] Eliane Magdalon, Stella Michaelsen, Antonio Quevedo, and Mindy Levin. 2011. Comparison of grasping movements made by healthy subjects in a 3-dimensional immersive virtual versus physical environment. Acta psychologica 138 (06 2011), 126-34. DOI : http://dx.doi.org/10.1016/j.actpsy.2011.05.015

[55] M. Nabiyouni, A. Saktheeswaran, D. A. Bowman, and A. Karanth. 2015. Comparing the performance of natural, semi-natural, and non-natural locomotion techniques in virtual reality. In 2015 IEEE Symposium on 3D User Interfaces (3DUI). 3-10. DOI:

http://dx.doi.org/10.1109/3DUI.2015.7131717

[56] Tao Ni, Doug A Bowman, Chris North, and Ryan P McMahan. 2011. Design and evaluation of freehand menu selection interfaces using tilt and pinch gestures. International Journal of Human-Computer Studies 69, 9 (2011), 551-562.

[57] Austin Nichols and Jon Maner. 2008. The Good-Subject Effect: Investigating Participant Demand Characteristics. The Journal of General Psychology 135 (05 2008), 151-65. DOI :

http://dx.doi.org/10.3200/GENP.135.2.151-166

[58] Ryuma Niiyama, Lining Yao, and Hiroshi Ishii. 2013. Weight and Volume Changing Device with Liquid Metal Transfer. In Proceedings of the 8th International Conference on Tangible, Embedded and Embodied Interaction (TEI '14). 49-52. DOI : http://dx.doi.org/10.1145/2540930.2540953

[59] Kristine Nowak and Frank Biocca. 2003. The Effect of the Agency and Anthropomorphism on Users' Sense of Telepresence, Copresence, and Social Presence in Virtual Environments. Presence Teleoperators amp Virtual Environments 12 (10 2003), 481-494. DOI: http://dx.doi.org/10.1162/105474603322761289

[60] Alex Peer and Kevin Ponto. 2017. Evaluating perceived distance measures in room-scale spaces using consumer-grade head mounted displays. In 2017 IEEE Symposium on 3D User Interfaces (3DUI). IEEE, 83-86.
[61] Roshan Lalitha Peiris, Yuan-Ling Feng, Liwei Chan, and Kouta Minamizawa. 2019. ThermalBracelet: Exploring Thermal Haptic Feedback Around the Wrist. In Proceedings of the 2019 CHI Conference on Human Factors in Computing Systems (CHI '19). ACM, New York, NY, USA, Article 170, 11 pages. DOI: http://dx.doi .org/10.1145/3290605.3300400

[62] Roshan Lalintha Peiris, Wei Peng, Zikun Chen, Liwei Chan, and Kouta Minamizawa. 2017. ThermoVR: Exploring Integrated Thermal Haptic Feedback with Head Mounted Displays. In Proceedings of the 2017 CHI Conference on Human Factors in Computing Systems (CHI '17). ACM, New York, NY, USA, 5452-5456. DOI :

http://dx.doi.org/10.1145/3025453.3025824

[63] Chao Peng, Jeffrey T. Hansberger, Lizhou Cao, and Vaidyanath A. Shanthakumar. 2017. Hand gesture controls for image categorization in immersive virtual environments. In 2017 IEEE Virtual Reality (VR). 331-332.

[64] Thies Pfeiffer. 2012. Using virtual reality technology in linguistic research. In 2012 IEEE Virtual Reality Workshops (VRW). 83-84. DOI : http://dx.doi.org/10.1109/VR.2012.6180893

[65] Jayesh S. Pillai, Azif Ismail, and Herold P. Charles. 2017. Grammar of VR Storytelling: Visual Cues. In Proceedings of the Virtual Reality International Conference - Laval Virtual 2017 (VRIC '17). Article 7, 4 pages. DOI: http://dx.doi.org/10.1145/3110292.3110300

[66] Thammathip Piumsomboon, Adrian Clark, Mark Billinghurst, and Andy Cockburn. 2013. User-defined gestures for augmented reality. In CHI'13 Extended Abstracts on Human Factors in Computing Systems. 955-960.

[67] Mores Prachyabrued and Christoph W Borst. 2016. Design and evaluation of visual interpenetration cues in virtual grasping. IEEE Transactions on Visualization and Computer Graphics 22, 6 (2016), 1718-1731.

[68] Angie Hill Price, Mathew Kuttolamadom, and Suleiman Obeidat. 2019. Using Virtual Reality Welding to Improve Manufacturing Process Education. (2019).

[69] Steffen Puhlmann, Fabian Heinemann, Oliver Brock, and Marianne Maertens. 2016. A compact representation of human single-object grasping. In 2016 IEEE/RSJ International Conference on Intelligent Robots and Systems (IROS). 1954-1959. DOI : http://dx.doi.org/10.1109/IROS. 2016.7759308

[70] Andreas Pusch, Olivier Martin, and Sabine Coquillart. 2008. HEMP-Hand-Displacement-Based Pseudo-Haptics: A Study of a Force Field Application. 2008 IEEE Symposium on 3D User Interfaces (2008), 59-66. 
[71] Andreas Pusch, Olivier Martin, and Sabine Coquillart. 2009. HEMP-hand displacement-based pseudo-haptics: A study of a force field application and a behavioural analysis. International Journal of Human-Computer Studies 67, 3 (2009), 256 - 268. DOI :

http://dx.doi.org/https: //doi.org/10.1016/j.ijhcs.2008.09.015 Current trends in 3D user interface research.

[72] Brittany Redmond, Rachel Aina, Tejaswi Gorti, and Blake Hannaford. 2010. Haptic Characteristics of Some Activities of Daily Living. In Proceedings of the 2010 IEEE Haptics Symposium (HAPTIC '10). IEEE Computer Society, 71-76. DOI : http://dx.doi.org/10.1109/HAPTIC.2010.5444674

[73] Gang Ren and Eamonn O’Neill. 2013. 3D selection with freehand gesture. Computers \& Graphics 37, 3 (2013), 101-120.

[74] Michael Rietzler, Florian Geiselhart, Jan Gugenheimer, and Enrico Rukzio. 2018. Breaking the Tracking: Enabling Weight Perception Using Perceivable Tracking Offsets. In Proceedings of the 2018 CHI Conference on Human Factors in Computing Systems (CHI'18). ACM, New York, NY, USA, Article 128, 12 pages. DOI : http://dx.doi.org/10.1145/3173574.3173702

[75] Katja Rogers, Jana Funke, Julian Frommel, Sven Stamm, and Michael Weber. 2019. Exploring Interaction Fidelity in Virtual Reality: Object Manipulation and Whole-Body Movements. In Proceedings of the 2019 CHI Conference on Human Factors in Computing Systems (CHI '19). ACM, New York, NY, USA, Article 414, 14 pages. DOI :

http://dx.doi.org/10.1145/3290605.3300644

[76] Javier Romero, Hedvig Kjellström, Carl Henrik Ek, and Danica Kragiç. 2013. Non-parametric hand pose estimation with object context. Image and Vision Computing 31, 8 (2013), 555 - 564.

http://www. sciencedirect.com/science/article/pii/ S0262885613000656

[77] Pedro Rosas, Felix Wichmann, and Johan Wagemans. 2007. Texture and object motion in slant discrimination: Failure of reliability-based weighting of cues may be evidence for strong fusion. Journal of vision 7 (04 2007), 3. DOI : http://dx.doi .org/10.1167/7.6.3

[78] Beatriz Sousa Santos, João Cardoso, Beatriz Quintino Ferreira, Carlos Ferreira, and Paulo Dias. 2016. Developing 3D Freehand Gesture-Based Interaction Methods for Virtual Walkthroughs: Using an Iterative Approach. In Handbook of Research on Human-Computer Interfaces, Developments, and Applications. IGI Global, 52-72.

[79] Luisa Sartori, Elisa Straulino, and Umberto Castiello. 2011. How Objects Are Grasped: The Interplay between Affordances and End-Goals. PLOS ONE 6, 9 (09 2011), 1-10. DOI :

http://dx.doi.org/10.1371/journal.pone.0025203

[80] Peter Schulz, Dmitry Alexandrovsky, Felix Putze, Rainer Malaka, and Johannes Schöning. 2019. The Role of Physical Props in VR Climbing Environments. DOI : http://dx.doi.org/10.1145/3290605.3300413

[81] Valentin Schwind, Pascal Knierim, Nico Haas, and Niels Henze. 2019. Using Presence Questionnaires in Virtual Reality. In Proceedings of the 2019 CHI Conference on Human Factors in Computing Systems (CHI'19). ACM, New York, NY, USA, Article 360, 12 pages. DOI : http://dx.doi.org/10.1145/3290605.3300590

[82] Valentin Schwind, Pascal Knierim, Cagri Tasci, Patrick Franczak, Nico Haas, and Niels Henze. 2017. "These Are Not My Hands!": Effect of Gender on the Perception of Avatar Hands in Virtual Reality. In Proceedings of the 2017 CHI Conference on Human Factors in Computing Systems (CHI'17). ACM, New York, NY, USA, 1577-1582. DOI :

http://dx.doi.org/10.1145/3025453.3025602

[83] Valentin Schwind, Sven Mayer, Alexandre Comeau-Vermeersch, Robin Schweigert, and Niels Henze. 2018. Up to the Finger Tip: The Effect of Avatars on Mid-Air Pointing Accuracy in Virtual Reality. In Proceedings of the 2018 Annual Symposium on Computer-Human Interaction in Play (CHI PLAY'18). ACM, New York, NY, USA, 477-488. DOI : http://dx.doi.org/10.1145/3242671.3242675

[84] Andrea Serino, Adrian Alsmith, Marcello Costantini, Alisa Mandrigin, Ana Tajadura-Jimenez, and Christophe Lopez. 2013. Bodily ownership and self-location: Components of bodily self-consciousness.

Consciousness and Cognition 22, 4 (2013), 1239 - 1252. DOI : http://dx.doi .org/https:

//doi.org/10.1016/j.concog.2013.08.013

[85] Samuel S. Shapiro and Martin B. Wilk. 1965. An analysis of variance test for normality (complete samples). Biometrika 52, 3/4 (1965), 591-611.

[86] David Somers and R. McNally. 2010. Kinesthetic visual capture induced by apparent motion. Journal of Vision J VISION 3 (10 2010), 35-35. DOI : http://dx.doi.org/10.1167/3.9.35

[87] Sota Suzuki, Haruto Suzuki, and Mie Sato. 2014. Grasping a Virtual Object with a Bare Hand. In ACM SIGGRAPH 2014 Posters (SIGGRAPH'14). ACM, New York, NY, USA, Article 51, 1 pages. DOI : http://dx.doi.org/10.1145/2614217.2630574

[88] Anthony Talvas, Maud Marchal, Christian Duriez, and Miguel A Otaduy. 2015. Aggregate constraints for virtual manipulation with soft fingers. IEEE transactions on visualization and computer graphics 21, 4 (2015), $452-461$.

[89] Jordan Tewell, Jon Bird, and George R. Buchanan. 2017a. The Heat is On: A Temperature Display for Conveying Affective Feedback. In Proceedings of the 2017 CHI Conference on Human Factors in Computing Systems (CHI'17). ACM, New York, NY, USA, 1756-1767. DOI : http://dx.doi.org/10.1145/3025453.3025844 
[90] Jordan Tewell, Jon Bird, and George R. Buchanan. 2017b. Heat-Nav: Using Temperature Changes As Navigation Cues. In Proceedings of the 2017 CHI Conference on Human Factors in Computing Systems (CHI '17). ACM, New York, NY, USA, 1131-1135. DOI : http://dx.doi.org/10.1145/3025453.3025965

[91] Aurelijus Vaitkevičius, Mantas Taroza, Tomas Blažauskas, Robertas Damaševičius, Rytis Maskeliūnas, and Marcin Wožniak. 2019. Recognition of American Sign Language Gestures in a Virtual Reality Using Leap Motion. Applied Sciences 9, 3 (2019). DOI:

http://dx.doi.org/10.3390/app9030445

[92] Antonin Viau, Anatol Feldman, Bradford McFadyen, and Mindy Levin. 2005. Reaching in reality and virtual reality: A comparison of movement kinematics in healthy subjects and in adults with hemiparesis. Journal of neuroengineering and rehabilitation 1 (01 2005), 11. DOI : http://dx . doi .org/10.1186/1743-0003-1-11

[93] Toinon Vigier, Guillaume Moreau, and Daniel Siret. 2015. From visual cues to climate perception in virtual urban environments. In 2015 IEEE Virtual Reality (VR). 305-306. DOI :

http://dx.doi.org/10.1109/VR.2015.7223417

[94] Daniel Vogel and Ravin Balakrishnan. 2005. Distant Freehand Pointing and Clicking on Very Large, High Resolution Displays. In Proceedings of the 18th Annual ACM Symposium on User Interface Software and Technology (UIST '05). 33-42. DOI : http://dx.doi.org/10.1145/1095034.1095041

[95] Emanuel Vonach, Clemens Gatterer, and Hannes Kaufmann. 2017. VRRobot: Robot actuated props in an infinite virtual environment. In 2017 IEEE Virtual Reality (VR). 74-83. DOI :

http://dx.doi.org/10.1109/VR.2017.7892233

[96] Supachai Vorapojpisut, Kevin Hillairet, Artit Boriboonsak, and Phongsit Misa. 2016. A Myo Armband-based Measurement Platform for Hand Rehabilitation Applications. In Proceedings of the International Convention on Rehabilitation Engineering \& Assistive Technology (i-CREATe 2016). Article 19, 4 pages. http://dl.acm.org.ezproxy.bcu.ac.uk/citation. cfm?id=3014393. 3014417

[97] Juan Pablo Wachs, Mathias Kölsch, Helman Stern, and Yael Edan. 2011. Vision-based hand-gesture applications. Commun. ACM 54, 2 (2011), 60-71.

[98] Robert Whitwell, Tzvi Ganel, Caitlin Byrne, and Melvyn Goodale. 2015. Real-time vision, tactile cues, and visual form agnosia in pantomimed grasping: Removing haptic feedback induces a switch from natural to pantomime like grasps. Front. Hum. Neurosci. 9 (01 2015).

[99] Raphael Wimmer. 2011. Grasp Sensing for Human-computer Interaction. In Proceedings of the Fifth International Conference on Tangible, Embedded, and Embodied Interaction (TEI '11). ACM, New York,
NY, USA, 221-228. DOI :

http://dx.doi.org/10.1145/1935701.1935745

[100] Bob G. Witmer and Michael J. Singer. 1998. Measuring Presence in Virtual Environments: A Presence Questionnaire. Presence 7, 3 (June 1998), 225-240. DOI :

http://dx.doi.org/10.1162/105474698565686

[101] Jacob O Wobbrock, Meredith Ringel Morris, and Andrew D Wilson. 2009. User-defined gestures for surface computing. In Proceedings of the SIGCHI Conference on Human Factors in Computing Systems. ACM, 1083-1092.

[102] Seahwa Won and Stephen Westland. 2017. Colour meaning and context. Color Research \& Application 42, 4 (2017), 450-459. DOI : http://dx.doi.org/10.1002/col.22095

[103] Yuan Yao, Po-Tsung Chiu, and Wai-Tat Fu. 2017. A Gestural Interface for Practicing Children's Spatial Skills. In Proceedings of the 22Nd International Conference on Intelligent User Interfaces Companion (IUI'17 Companion). ACM, 43-47.

[104] Dianna Yim, Garance Nicole Loison, Fatemeh Hendijani Fard, Edwin Chan, Alec McAllister, and Frank Maurer. 2016. Gesture-driven Interactions on a Virtual Hologram in Mixed Reality. In Proceedings of the 2016 ACM Companion on Interactive Surfaces and Spaces (ISS Companion '16). ACM, 55-61.

[105] André Zenner and Antonio Krüger. 2017. Shifty: A Weight-Shifting Dynamic Passive Haptic Proxy to Enhance Object Perception in Virtual Reality. IEEE Transactions on Visualization and Computer Graphics 23, 4 (April 2017), 1285-1294.

[106] Yingwei Zhang, Yiqiang Chen, Hanchao Yu, Xiaodong Yang, Wang Lu, and Hong Liu. 2018.

Wearing-independent Hand Gesture Recognition Method Based on EMG Armband. Personal Ubiquitous Comput. 22, 3 (June 2018), 511-524. DOI :

http://dx.doi.org/10.1007/s00779-018-1152-3

[107] Yun Zhou, Shangpeng Ji, Tao Xu, and Zi Wang. 2018. Promoting Knowledge Construction: A Model for Using Virtual Reality Interaction to Enhance Learning.

Procedia Computer Science 130 (2018), 239 - 246. DOI : http://dx.doi.org/https:

//doi.org/10.1016/j.procs.2018.04.035 The 9th International Conference on Ambient Systems, Networks and Technologies (ANT 2018) / The 8th International Conference on Sustainable Energy Information Technology (SEIT-2018) / Affiliated Workshops.

[108] Mounia Ziat, Taylor Rolison, Andrew Shirtz, Daniel Wilbern, and Carrie Anne Balcer. 2014. Enhancing virtual immersion through tactile feedback. In Proceedings of the adjunct publication of the 27th annual ACM symposium on User interface software and technology. 65-66. 